effective only in 2; IV immunoglobulin was ineffective in all 13 patients treated. Followup ranged from 8-194 months (mean 60.9 months), and outcome was uniformly poor. All had residual epilepsy without a latent period, and the majority had cognitive impairment. WISC scores were $<70$ in 16 and $<20$ in 10 patients. All with GluRe2 antibodies were cognitively impaired. Other neurologic deficits included memory impairment, autistic tendency, hyperkinesis, learning disability, and emotional instability, each occurring in $15-22 \%$ cases. One patient died of unknown cause at 9 year after onset. (Sakuma H, Awaya Y, Shiomi M, et al. Acute encephalitis with refractory, repetitive partial seizures (AERRPS): a peculiar form of childhood encephalitis. Acta Neurol Scand April 2010;121:251-256). (Respond: Dr H Sakuma, National Center of Neurology and Psychiatry, Tokyo, Japan. E-mail: sakumh@ncnp.go.jp).

COMMENT. AERRPS differs from most types of childhood encephalitis with post-encephalitic epilepsy in that intractable partial seizures develop without a latent period between the acute febrile phase and the onset of epilepsy. Awaya Y and Fukuyama Y (1986) first described this unusual form of encephalitis and postencephalitic epilepsy in 5 cases. More than 30 cases have now been reported in Japan (Awaya et al. 2007). Similar cases have also been reported worldwide. The cause is unknown but an autoimmune process related to anti-GluRe2 antibodies may be contributory.

\title{
ANTI-N-METHYL-D-ASPARTATE RECEPTOR ENCEPHALITIS: RESPONSE TO EARLY TREATMENT
}

A 5-year-old girl with anti-N-methyl-D-aspartate (NMDA) receptor encephalitis and near-complete recovery following early diagnosis and treatment with immune globulin and steroids is reported from Indiana University, University of Pennsylvania, and Mayo Clinic. She presented with episodic headache, paresthesias and weakness of left hand, agitation, and slurred speech. CT scan, MRI, and video-EEG were initially normal. Symptoms rapidly progressed with choreoathetoid movements, urinary incontinence, mutism, rhythmic tongue thrusting, and dysphagia. EEG on day 3 showed bilateral slowing and occipital epileptiform discharges. Methylprednisolone (IV, 40 $\mathrm{mg} / \mathrm{kg} /$ day) for 4 days was followed by improvement that plateaued. IV Octagam $(0.4$ $\mathrm{g} / \mathrm{kg} /$ day) was given for 5 days, and oral steroids were tapered over 12 weeks. At 5 -week follow-up, recovery was almost complete. Immunohistochemical analysis (Mayo Clinic) of CSF obtained on admission revealed anti-NMDA antibodies, with molecular confirmation at University of Pennsylvania. Pelvic MRI was unremarkable, but close observation was advised to rule out relapse and occult ovarian teratoma. (Breese EH, Dalmau J, Lennon VA, Apiwattanakul M, Sokol DK. Anti-N-methyl-D-aspartate receptor encephalitis: early treatment is beneficial. Pediatr Neurol 2010;42:213-214). (Respond: Dr Sokol, Riley Hospital for Children, Indiana University School of Medicine, 575 West Drive, Indianapolis, IN 46202. E-mail: dksokol@iupui.edu).

COMMENT. Specific antibody detection is indicated in young children presenting with acute psychiatric and movement disorders such as agitation and dyskinesias. Ovarian teratoma associated with NMDAR encephalitis in young female 
adults is uncommon in children, but pelvic imaging (MRI) is important since surgical removal of a tumor is followed by rapid recovery. See Ped Neur Briefs Jan 2010;24:1-2, for reports of NMDAR and dyskinetic encephalitis lethargica (Dale RC et al. Ann Neurol 2009;66:704-709). In the above case report, pelvic MRI was negative, but the authors recommend close surveillance. They credit the favorable outcome to early initiation of immunosuppressant therapy.

\section{MOVEMENT DISORDERS}

\section{OPSOCLONUS-MYOCLONUS FOLLOWING MYCOPLASMA PNEUMONIAE INFECTION}

Three adolescent cases of opsoclonus-myoclonus (OMS) that followed infection with mycoplasma pneumoniae are reported from University Hospital, Bern, Switzerland. Case 1. A 10-year-old girl presented with a 3-day history of uncontrolled eye movements and whole body tremor, followed by cerebellar ataxia. Two weeks previously she had pneumonia due to $M$ pneumoniae (nasopharyngeal PCR positive, and IgM antibodies elevated). MRI brain showed no signs of inflammation, and tests for a paraneoplastic etiology were negative. Following methylprednisolone, symptoms slowly improved. Steroids were weaned over 10 months, and at 30-month follow-up, no relapse had occurred. Two further cases, both aged 14 years, developed OMS 2 to 4 weeks following $M$ pneumoniae respiratory infection. Neuroblastoma was ruled out. Recovery from OMS occurred after 2 to 4 weeks of methylprednisolone therapy. (Huber BM, Strozzi S, Steinlin M, Aebi C, Fluri S. Mycoplasma pneumoniae associated opsoclonus-myoclonus syndrome in three cases. Eur J Pediatr April 2010;169:441-445). (Respond: Dr S Fluri, Department of Pediatrics, University Hospital, Inselspital, CH-3010 Bern, Switzerland. E-mail: simon.fluri@insel.ch).

COMMENT. One previous case of OMS following $M$ pneumoniae infection is cited in a 4-year-old girl (Chemli J et al. Arch Pediatr 2007;14:1003-1006). Among pediatric cases, OMS generally presents between 6 months and 3 years of age, and neuroblastoma is present in $50 \%$ cases. In the above parainfectious childhood cases of OMS, response to steroid therapy was rapid and generally complete, and the long-term outcome was favorable. Neurological complications of $M$ pneumoniae include meningoencephalitis, acute disseminated encephalomyelitis, acute transverse myelitis, cerebellitis, abducens nerve and Bell's palsies, and Guillain Barre syndrome. (Yis U et al. Eur J Pediatr 2008;167(9):973-978).

\section{SEIZURE DISORDERS}

\section{ANTIHISTAMINES AND FEBRILE SEIZURE SUSCEPTIBILITY}

Charts of children with febrile seizures admitted to the Hospital of Shiga University of Medical Sciences, Otsu, Japan, 2006-2007, were reviewed retrospectively. Clinical characteristics of 23 patients who received antihistamines were compared with 